\title{
Active Surveillance of Carbapenem-resistant Gram- negative Bacteria to guide antibiotic therapy: a single-center prospective observational study
}

\section{Qiqiang Liang}

Zhejiang University School of Medicine Second Affiliated Hospital

\section{Juan Chen}

Zhejiang University School of Medicine Second Affiliated Hospital

\section{Yongshan Xu}

Zhejiang University School of Medicine Second Affiliated Hospital

\section{Yibing Chen}

Zhejiang University School of Medicine Second Affiliated Hospital

Man Huang ( $\nabla$ huangman@zju.edu.cn )

Zhejiang University School of Medicine https://orcid.org/0000-0002-0842-2254

\section{Research}

Keywords: Carbapenem-resistant Gram-negative Bacteria, Active Surveillance, Empirical antibiotic therapy, Infection after colonization, Nosocomial infection prevention and control

Posted Date: September 13th, 2021

DOl: https://doi.org/10.21203/rs.3.rs-889531/v1

License: (c) (1) This work is licensed under a Creative Commons Attribution 4.0 International License. Read Full License 


\section{Abstract \\ Background}

Carbapenem-resistant Gram-negative Bacteria (CRGNB) have become a public health concern worldwide. The risk factors associated with CRGNB infection after colonization are unknown, nor is the optimal timing of antibiotic treatment, warranting further investigation.

\section{Methods}

A 4-year single-center prospective observational study was conducted. CRGNB-colonized patients were incorporated on admission into our observation cohort for an active surveillance culture program, and analysis of risk factors associated with infections after CRGNB colonization was performed. We divided patients into empirical antibiotic therapy groups and standard antibiotic therapy groups according to whether antibiotics were used before or after cultures yielded a result to explore the relationship between the timing of antibiotics and clinical efficacy.

\section{Results}

152 out of 451 CRGNB-colonized patients in the prospective observational cohort developed CRGNB infection. The risk factors associated with CRGNB infection after colonization included CRKP $(P<0.001$, $\mathrm{OR}=3.27)$ and $\mathrm{CRPA}(P<0.001, \mathrm{OR}=2.97)$ colonization, history of carbapenems use $(P<0.001, \mathrm{OR}=$ $5.48)$, and immunocompromise $(P<0.001, \mathrm{OR}=7.07)$. There were 88 infected patients in the empirical antibiotic therapy groups and 64 in standard antibiotic therapy groups. The mortality was lower in empirical therapy groups than standard therapy groups $(17.0 \%$ vs. $37.5 \%, P=0.004, \mathrm{OR}=0.32)$.

\section{Conclusions}

CRGNB colonization increased the risk of infection, and risk factors included CRKP and CRPA colonization, immunocompromise, and prior carbapenems use. Once infection occurs in CRGNBcolonized patients, susceptibility-guided antibiotic treatment can be given according to previous susceptibility results of colonized CRGNB, reducing mortality.

\section{Background:}

Carbapenem-resistant Gram-negative bacteria (CRGNB) are one of the most serious multidrug-resistant bacteria (MDR) globally, accounting for a significant portion of Hospital Acquired Infections (HAl) and are associated with increased mortality and prolonged hospitalizations $(1,2)$. Active surveillance for early recognition of CRGNB carriers and other infection control measures, including contact isolation and decontamination strategies, are essential to reduce the spread of $\operatorname{MDR}(3,4)$. The present study found that 
active surveillance combined with early or preemptive isolation could reduce the spread of carbapenemresistant enterobacteria (CRE), decreasing carriage rates from 9.8-7.6\%, and infection rates from 5.2$2.8 \%(5)$. A meta-analysis of 10 observational studies demonstrated CRE-colonized patients had a $16.5 \%$ risk of infection. Recent studies have demonstrated that the infection rates after CRGNB colonization ranged from $11-30 \%$ and were influenced by CRGNB endemicity and the implementation of IPC strategies(6).

Stringent measures have been designed to block the spread of CRE infection, encompassing active surveillance, strict contact isolation and hand hygiene, selective digestive decontamination (SDD), and restricted use of carbapenem antibiotics $(4,7)$. Antibiotics such as polymyxin B and ceftazidimeavibactam are acknowledged for their efficiency against CRGNB, while other measures have been developed, including the combination of antibiotics (double carbapenem combinations), prolonged infusion of carbapenem, and inhalation of polymyxin B and aminoglycosides to provide more options for treatment of CRGNB infections(8-12). Nowadays, guidelines recommend early empirical antibiotic treatments for severe infections but tend to be cautious for the optimal antibiotic timing in CRGNB infection because of the paucity of effective antibiotics and potential inferior efficacy of the "old" antibiotics.

Antibiotics are usually indicated after CRGNB infection with documentation by culture yields, which may delay treatment for at least 2-3 days. Some studies have pointed out that earlier use of sensitive antibiotics may reduce the mortality of CRE bloodstream infection; however, there is a risk that indiscriminate use of antibiotics makes the pathogen more resistant, leading to antibiotic resistance(13). Based on our experience, it seems more reasonable to use susceptibility-guided antibiotic treatment in CRGNB-colonized patients, but there is little empirical evidence. The purpose of this study was to explore the risk factors of CRGNB infection after colonization by active surveillance and to analyze the clinical effects of susceptibility-guided antibiotic treatment.

\section{Methods:}

1. Study design:

This study was a single-center prospective observational study conducted at the General Intensive Care Unit (ICU) of the Second Affiliated Hospital of Zhejiang University, a 1200-bed tertiary academic care hospital with 40 intensive care beds in Hangzhou China. The study period spanned from 2017.01.01 to 2020.12.31. Since 2017.01, our ICU has implemented robust infection prevention and control (IPC) measures against $\mathrm{CRE}$, emphasizing active surveillance combined with early or preemptive isolation. We have adopted active surveillance and individual isolation for patients with a high risk of MDR carriage, with two negative tests prerequisites for release from isolation(5). In this study, CRGNB-colonized patients in active surveillance at admission were incorporated into the observation cohort. The incidence of infections after colonization and potential risk factors were analyzed. Then, we assessed the relationship between the timing of antibiotics use and patient mortality. This study was conducted after approval 
from the ethics committee of the Second Affiliated Hospital of Zhejiang University school of medicine (Batch number IRB-2016-1511).

\section{Data definition:}

2.1 Inclusion criteria: Patients who underwent active surveillance on admission with any positive surveillance culture from the throat, rectal, or inguinal swabs, or other specimens sent by the clinicians. Inclusion criteria for active surveillance:

(1) Patients transferred from other medical institutions, including (a) Patients hospitalized for more than seven days; (b) Patients hospitalized for less than seven days but who were in contact with suspected CRE patients or symptomatic; (c) Patients with prolonged hospitalization in private rehabilitation hospitals and nursing homes; (d) CRE-infected patients who were transferred from general wards but were hospitalized for less than three days.

(2) Symptomatic patients transferred from high-risk general wards (a high-risk ward was defined as a ward with patients suffering from CRE infection within the preceding three months according to the hospital antimicrobial resistance monitoring report);

(3) Patients who had been confirmed to be CRE carriers within the past year.

2.2 Exclusion criteria: Diagnosis of CRGNB infection on admission, admission less than 48 hours, and age under 16 . Diagnosis of CRGNB infection was made by two senior attending physicians independently. Diagnosis of Bloodstream infection was made in the presence of clinical manifestations in patients with positive blood cultures. Diagnosis of Hospital-Acquired Pneumonia was based on the 2016 USA IDS/ATS guidelines(14) and Urinary tract infection on the 2009 USA IDS guidelines(15).

2.3. Empirically sensitive antibiotic therapy was given to CRGNB-colonized patients diagnosed with CRGNB infection, and before the culture yielded a result, antibiotic selection (ceftazidime-avibactam, colistin, and tigecycline) was based on the previous (colonization) CRGNB susceptibility results. Standard antibiotic therapy groups were given to CRGNB-colonized patients diagnosed with CRGNB infection, and antibiotic selection was based on the CRGNB susceptibility results after the cultures yielded results.

2.4. Other important definitions: Medication history was limited to 3 months before CRGNB colonization, including cephalosporins, carbapenems, oral or intravenous glucocorticoids. Invasive procedures included catheterizations of deep veins and arteries for continuous renal replacement therapy, pulse indicator continuous cardiac output, and extracorporeal membrane oxygenation. Immunocompromised patients included patients with chemotherapy and long-term use of glucocorticoids or immunosuppressants. Other department history refers to patients who were admitted to other departments for more than three days. Other hospital history refers to the hospitalization in other hospitals or nursing institutions in the past six months. The definition of Acute kidney injury was based on the guidelines of The Kidney Disease: Improving Global Outcomes(16). 
3. Microbiological tests: CRGNB strains including CRE, carbapenem-resistant Klebsiella pneumoniae (CRKP), carbapenem-resistant Pseudomonas aeruginosa (CRPA), and carbapenem-resistant

Acinetobacter baumannii (CRAB), were resistant to at least one of the carbapenems, including imipenem, meropenem, and ertapenem. The Antimicrobial susceptibility tests were performed with automated microbial identification and drug susceptibility systems (VITEK2 AST-GN16 France). Minimum inhibitory concentration (MIC) determination and interpretation complied with standards established by the Clinical and Laboratory Standards Institute (CLSI). Resistance to carbapenem was defined as a MIC $\geq 2 \mathrm{mg} / \mathrm{L}$ for imipenem or meropenem according to CLSI guidelines. Tigecycline resistance was interpreted according to the breakpoints defined by The European Committee on Antimicrobial Susceptibility Testing (MIC>2 $\mathrm{mg} / \mathrm{L})$.

4. Statistical methods: all statistical analyses were performed in R and RStudio. In univariate analysis, numerical variables were tested by independent sample T-test, dichotomous variables were compared by chi-square test, and a $P$ value less than 0.05 was considered statistically significant. Odds ratio (OR) and $95 \%$ confidence interval $(\mathrm{Cl})$ were calculated to evaluate the strength of association.

\section{Results:}

From 2017.01.01 to 2020.12.31, our center treated a total of 6645 patients with 3754 cases that underwent active surveillance. 548 (14.6\%) patients yielded positive CRGNB surveillance cultures, and 97 cases presented with CRGNB infection on admission. Finally, 451 (12.0\%) CRGNB-colonized patients were included, among which 152 (33.7\%) developed CRGNB infection. There was no significant difference in gender, age, primary disease, acute physiology and chronic health evaluation (APACHE) II scores, and distribution of surveillance swab sites between the CRGNB infection and control groups. Surveillance cultures with the greatest proportion of positive yields came from throat swabs and sputum, followed by rectal swabs, inguinal swabs, and feces. Interestingly, the proportion of CRKP and CRPA was higher in the infection group, while CRAB was more common in the control group. The relationship between the distribution of swab sites, bacterial type, infection or not, or colonization and outcome is shown in Fig. 1. Compared with the control group, infected patients were associated with prior use of carbapenems (46.7\% vs. $14.7 \%, \mathrm{P}<0.001, \mathrm{OR}=5.08 .95 \% \mathrm{Cl}: 3.23-7.97)$ and glucocorticoids $(28.9 \%$ vs. $15.7 \%, \mathrm{P}=$ $0.001, \mathrm{OR}=2.18,95 \% \mathrm{Cl}: 1.36-3.49)$ use, and invasive procedures $(87.5 \% \mathrm{vs} .76 .3 \%, \mathrm{P}=0.005, \mathrm{OR}=2.18$, $95 \% \mathrm{Cl}: 1.26-3.78)$. Moreover, patients in the infection group were more likely to be immunocompromised (47.4\% vs. $23.1 \%, \mathrm{P}<0.001, \mathrm{OR}=3.00,95 \% \mathrm{Cl}$ : $1.98-4.55)$, previously hospitalized in another center (59.2\% vs. $49.2 \%, \mathrm{P}=0.043, \mathrm{OR}=1.52,95 \% \mathrm{Cl}: 1.01-2.28)$ and previously admitted in another department (34.2\% vs. $22.4 \%, \mathrm{P}=0.007, \mathrm{OR}=1.80,95 \% \mathrm{Cl}: 1.17-2.77)$ than the control group. The incidence of septic shock (55.2\% vs. $27.1 \%, \mathrm{P}<0.001, \mathrm{OR}=3.32,95 \% \mathrm{Cl}$ : $2.21-5.00)$ and $\mathrm{AKI}(48.7 \%$ vs. $23.1 \%, \mathrm{P}<0.001, \mathrm{OR}=$ $3.16,95 \% \mathrm{Cl}: 2.08-4.79)$ were higher in the infection group, with longer ICU stay, hospital stay and higher mortality $(25.6 \%$ vs. $14.0 \%, P=0.002, O R=2.11,95 \% \mathrm{Cl}: 1.30-3.44)$ than the control group.

Furthermore, we identified six risk factors of CRGNB infection after colonization by multiple regression analysis, including CRKP colonization $(\mathrm{P}<0.001, \mathrm{OR}=3.27,95 \% \mathrm{Cl}: 1.80-5.95)$, CRPA colonization $(\mathrm{P}<$ 
$0.001, \mathrm{OR}=2.97,95 \% \mathrm{Cl}: 1.63-5.40)$, prior admission in other departments $(\mathrm{P}=0.029, \mathrm{OR}=1.78,95 \% \mathrm{Cl}$ : 1.06-2.97), invasive procedure $(P<0.001, O R=6.22,95 \% \mathrm{Cl} \otimes 2.57-15.03)$, history of carbapenem use $(P<$ $0.001, \mathrm{OR}=5.48,95 \% \mathrm{Cl}: 3.27-9.19)$, and immunocompromise $(\mathrm{P}<0.001, \mathrm{OR}=7.07,95 \% \mathrm{Cl}: 3.90-12.80)$. More details are shown in Table 2. 
Table 1

Baseline characteristic of the CRGNB colonized patients at active surveillance

\begin{tabular}{|c|c|c|c|c|}
\hline & $\begin{array}{l}\text { Infection after colonization, } \\
N=152\end{array}$ & $\begin{array}{l}\begin{array}{l}\text { No } \\
\text { infection, }\end{array} \\
N=299\end{array}$ & $\mathbf{P}$ & OR $(95 \% \mathrm{Cl})$ \\
\hline Age, yr, mean & 60.6 & 59.0 & 0.32 & / \\
\hline Male sex, no. (\%) & $112(73.6 \%)$ & $\begin{array}{l}206 \\
(68.9 \%)\end{array}$ & 0.29 & $\begin{array}{l}0.85(0.51- \\
1.22)\end{array}$ \\
\hline \multicolumn{5}{|l|}{ Primary disease (\%) } \\
\hline $\begin{array}{l}\text { Cerebrovascular accidents, } \\
\text { no. }(\%)\end{array}$ & $38(25.0 \%)$ & $\begin{array}{l}79 \\
(26.4 \%)\end{array}$ & 0.74 & $\begin{array}{l}0.93(0.59- \\
1.45)\end{array}$ \\
\hline Cardiac insufficiency, no. (\%) & $16(10.5 \%)$ & $26(8.7 \%)$ & 0.52 & $\begin{array}{l}1.24(0.64- \\
2.38)\end{array}$ \\
\hline Trauma, no. (\%) & $61(40.1 \%)$ & $\begin{array}{l}115 \\
(38.5 \%)\end{array}$ & 0.75 & $\begin{array}{l}1.07(0.72- \\
1.59)\end{array}$ \\
\hline Infection, no. (\%) & $49(32.2 \%)$ & $\begin{array}{l}82 \\
(27.4 \%)\end{array}$ & 0.28 & $\begin{array}{l}1.25(0.82- \\
1.92)\end{array}$ \\
\hline Malignant tumor, no. (\%) & $11(7.2 \%)$ & $26(8.7 \%)$ & 0.59 & $\begin{array}{l}0.82(0.39- \\
1.71)\end{array}$ \\
\hline Diabetes mellitus, no. (\%) & $14(9.2 \%)$ & $\begin{array}{l}41 \\
(13.7 \%)\end{array}$ & 0.17 & $\begin{array}{l}0.64(0.34- \\
1.21)\end{array}$ \\
\hline APACHE II scores (median) & 16.8 & 16.4 & 0.29 & / \\
\hline $\begin{array}{l}\text { Intubation or tracheotomy, } \\
\text { no. (\%) }\end{array}$ & $76(50.0 \%)$ & $\begin{array}{l}162 \\
(54.2 \%)\end{array}$ & 0.40 & $\begin{array}{l}0.86(0.57- \\
1.25)\end{array}$ \\
\hline $\begin{array}{l}\text { Prior cephalosporins history, } \\
\text { no. }(\%)\end{array}$ & $116(76.35)$ & $\begin{array}{l}198 \\
(66.2 \%)\end{array}$ & 0.028 & $\begin{array}{l}1.64(1.05- \\
2.56)\end{array}$ \\
\hline $\begin{array}{l}\text { Prior carbapenems history, } \\
\text { no. (\%) }\end{array}$ & $71(46.7 \%)$ & $\begin{array}{l}44 \\
(14.7 \%)\end{array}$ & $<0.001$ & $\begin{array}{l}5.08(3.23- \\
7.97)\end{array}$ \\
\hline Invasive operation, no. (\%) & $133(87.5 \%)$ & $\begin{array}{l}228 \\
(76.3 \%)\end{array}$ & 0.005 & $\begin{array}{l}2.18(1.26- \\
3.78)\end{array}$ \\
\hline Operation History, no. (\%) & $20(13.2 \%)$ & $29(9.7 \%)$ & 0.26 & $\begin{array}{l}1.41(0.76- \\
2.58)\end{array}$ \\
\hline $\begin{array}{l}\text { Glucocorticoid history, no. } \\
(\%)\end{array}$ & $44(28.9 \%)$ & $\begin{array}{l}47 \\
(15.7 \%)\end{array}$ & 0.001 & $\begin{array}{l}2.18(1.36- \\
3.49)\end{array}$ \\
\hline Immunocompromise, no. (\%) & $72(47.4 \%)$ & $\begin{array}{l}69 \\
(23.1 \%)\end{array}$ & $<0.001$ & $\begin{array}{l}3.00(1.98- \\
4.55)\end{array}$ \\
\hline Other hospital history, no. (\%) & $90(59.2 \%)$ & $\begin{array}{l}147 \\
(49.2 \%)\end{array}$ & 0.043 & $\begin{array}{l}1.52(1.01- \\
2.28)\end{array}$ \\
\hline
\end{tabular}




\begin{tabular}{|c|c|c|c|c|}
\hline & $\begin{array}{l}\text { Infection after colonization, } \\
N=152\end{array}$ & $\begin{array}{l}\begin{array}{l}\text { No } \\
\text { infection, }\end{array} \\
N=299\end{array}$ & $\mathbf{P}$ & OR $(95 \% \mathrm{Cl})$ \\
\hline $\begin{array}{l}\text { Another department history, } \\
\text { no. }(\%)\end{array}$ & $52(34.2 \%)$ & $\begin{array}{l}67 \\
(22.4 \%)\end{array}$ & 0.007 & $\begin{array}{l}1.80(1.17- \\
2.77)\end{array}$ \\
\hline \multicolumn{5}{|l|}{ CRGNB type, no. (\%) } \\
\hline CRKP & $60(39.5 \%)$ & $\begin{array}{l}80 \\
(26.7 \%)\end{array}$ & 0.006 & $\begin{array}{l}1.78(1.18- \\
2.70)\end{array}$ \\
\hline CRPA & $56(36.8 \%)$ & $\begin{array}{l}90 \\
(30.1 \%)\end{array}$ & 0.15 & $\begin{array}{l}1.35(0.89- \\
2.04)\end{array}$ \\
\hline CRAB & $32(21.1 \%)$ & $\begin{array}{l}102 \\
(34.1 \%)\end{array}$ & 0.004 & $\begin{array}{l}0.51(0.32- \\
0.84)\end{array}$ \\
\hline Other CRE & $4(2.6 \%)$ & $27(9.0 \%)$ & 0.025 & $\begin{array}{l}0.34(0.12- \\
0.90)\end{array}$ \\
\hline \multicolumn{5}{|l|}{ Active surveillance sites, no. (\%) } \\
\hline $\begin{array}{l}\text { Throat swab and/or sputum } \\
\text { culture }\end{array}$ & $115(75.6 \%)$ & $\begin{array}{l}213 \\
(71.2 \%)\end{array}$ & 0.31 & $\begin{array}{l}1.25(0.80- \\
1.96)\end{array}$ \\
\hline $\begin{array}{l}\text { Rectal swab and/or fecal } \\
\text { culture }\end{array}$ & $30(19.7 \%)$ & $\begin{array}{l}72 \\
(24.1 \%)\end{array}$ & 0.29 & $\begin{array}{l}0.77(0.48- \\
1.25)\end{array}$ \\
\hline Other cultures & $7(4.6 \%)$ & $14(4.7 \%)$ & 0.97 & $\begin{array}{l}0.98(0.38- \\
2.48)\end{array}$ \\
\hline Septic shock, no. (\%) & $84(55.2 \%)$ & $\begin{array}{l}81 \\
(27.1 \%)\end{array}$ & $<.001$ & $\begin{array}{l}3.32(2.21- \\
5.00)\end{array}$ \\
\hline Acute kidney injury, no. (\%) & $74(48.7 \%)$ & $\begin{array}{l}69 \\
(23.1 \%)\end{array}$ & $<.001$ & $\begin{array}{l}3.16(2.08- \\
4.79)\end{array}$ \\
\hline Death, no. (\%) & $39(25.6 \%)$ & $\begin{array}{l}42 \\
(14.0 \%)\end{array}$ & 0.002 & $\begin{array}{l}2.11(1.30- \\
3.44)\end{array}$ \\
\hline Length of ICU stay, day & 17.8 & 8.9 & $<001$ & / \\
\hline Length of hospital stay, day & 27.6 & 19.3 & $<001$ & / \\
\hline
\end{tabular}


Table 2

high-risk factors of infection after colonization in multivariate regression analysis

\begin{tabular}{|lll|}
\hline Variable & P & OR $(95 \% \mathrm{Cl})$ \\
\hline CRKP colonization & $<0.001$ & $3.27(1.80-5.95)$ \\
\hline CRPA colonization & $<0.001$ & $2.97(1.63-5.40)$ \\
\hline Another department history & 0.029 & $1.78(1.06-2.97)$ \\
\hline Invasive operation & $<0.001$ & $6.22(2.57-15.03)$ \\
\hline Carbapenems history & $<0.001$ & $5.48(3.27-9.19)$ \\
\hline Immunocompromise & $<0.001$ & $7.07(3.90-12.80)$ \\
\hline
\end{tabular}

Patients infected after colonization were divided into empirically sensitive antibiotic therapy groups $(\mathrm{n}=$ 88 ) and standard antibiotic therapy groups $(n=64)$, depending on the timing of antibiotic use. No statistical difference in basic characteristics was found between the two groups (Table 3 ). The incidence of bloodstream infection was higher in empirically therapy groups than standard therapy groups; however, no statistical differences were found for the remaining part of the infection and the bacterial types. The most common antibiotic therapy used was combination therapy based on tigecycline and colistin, followed by ceftazidime-avibactam monotherapy. The average interval between colonization and infection was around 13 days, and ICU hospitalization was about 16 days after CRGNB infection. The mortality was lower in the empirical therapy groups than standard therapy groups $(17.0 \%$ vs. $37.5 \%, \mathrm{P}=$ $0.004, \mathrm{OR}=0.32,95 \% \mathrm{Cl}: 0.16-0.73)$, which was substantiated by survival curve analysis $(P=0.002)$ (Fig. 2). 
Table 3

The timing and clinical efficacy of antibiotics for infected patients after colonization.

\begin{tabular}{|c|c|c|c|c|}
\hline & $\begin{array}{l}\text { Empirically sensitive } \\
\text { antibiotic therapy groups, } \mathrm{N} \\
=88\end{array}$ & $\begin{array}{l}\text { Standard antibiotic } \\
\text { therapy groups, } N=64\end{array}$ & $\mathbf{P}$ & $\begin{array}{l}\text { OR } \\
(95 \% \mathrm{Cl})\end{array}$ \\
\hline Age-yr & 61.2 & 59.8 & 0.60 & / \\
\hline Male sex, no. (\%) & $66(75.0 \%)$ & $46(71.8 \%)$ & 0.66 & $\begin{array}{l}0.85 \\
(0.41- \\
1.76)\end{array}$ \\
\hline $\begin{array}{l}\text { APACHE II scores } \\
\text { (median) }\end{array}$ & 17.0 & 16.4 & 0.20 & / \\
\hline $\begin{array}{l}\text { Cerebrovascular } \\
\text { accidents, no. (\%) }\end{array}$ & $22(25.0 \%)$ & $16(25.0 \%)$ & 1.00 & $\begin{array}{l}1.00 \\
(0.47- \\
2.10)\end{array}$ \\
\hline $\begin{array}{l}\text { Cardiac insufficiency, } \\
\text { no. (\%) }\end{array}$ & $9(10.2 \%)$ & $7(10.9 \%)$ & 0.88 & $\begin{array}{l}0.93 \\
(0.32- \\
2.63)\end{array}$ \\
\hline Trauma, no. (\%) & $38(43.2 \%)$ & $23(35.9 \%)$ & 0.36 & $\begin{array}{l}1.35 \\
(0.69- \\
2.62)\end{array}$ \\
\hline Infection, no. (\%) & $24(27.3 \%)$ & $25(39.1 \%)$ & 0.12 & $\begin{array}{l}0.58 \\
(0.29- \\
1.16)\end{array}$ \\
\hline $\begin{array}{l}\text { Malignant tumor, no. } \\
(\%)\end{array}$ & $9(10.2 \%)$ & $2(3.1 \%)$ & 0.09 & $\begin{array}{l}3.52 \\
(0.73- \\
16.94)\end{array}$ \\
\hline $\begin{array}{l}\text { Diabetes mellitus, no. } \\
(\%)\end{array}$ & $9(10.2 \%)$ & $5(7.8 \%)$ & 0.61 & $\begin{array}{l}1.34 \\
(0.42- \\
4.22)\end{array}$ \\
\hline $\begin{array}{l}\text { Intubation or } \\
\text { tracheotomy, no. (\%) }\end{array}$ & $45(51.1 \%)$ & $31(48.4 \%)$ & 0.74 & $\begin{array}{l}1.11 \\
(0.58- \\
2.12)\end{array}$ \\
\hline $\begin{array}{l}\text { Invasive operation, no. } \\
(\%)\end{array}$ & $72(81.8 \%)$ & $51(79.6 \%)$ & 0.74 & $\begin{array}{l}1.14 \\
(0.51- \\
2.59)\end{array}$ \\
\hline $\begin{array}{l}\text { Operation History, no. } \\
(\%)\end{array}$ & $10(11.3 \%)$ & $10(15.6 \%)$ & 0.44 & $\begin{array}{l}0.69 \\
(0.27- \\
1.77)\end{array}$ \\
\hline $\begin{array}{l}\text { Immunocompromise, } \\
\text { no. (\%) }\end{array}$ & $46(52.2 \%)$ & $26(40.6 \%)$ & 0.15 & $\begin{array}{l}1.60 \\
(0.83- \\
3.06)\end{array}$ \\
\hline Septic shock, no. (\%) & $44(50.0 \%)$ & $40(62.5 \%)$ & 0.12 & $\begin{array}{l}0.60 \\
(0.31- \\
1.15)\end{array}$ \\
\hline
\end{tabular}




\begin{tabular}{|c|c|c|c|c|}
\hline & $\begin{array}{l}\text { Empirically sensitive } \\
\text { antibiotic therapy groups, } \mathbf{N} \\
=\mathbf{8 8}\end{array}$ & $\begin{array}{l}\text { Standard antibiotic } \\
\text { therapy groups, } N=64\end{array}$ & $\mathbf{P}$ & $\begin{array}{l}\text { OR } \\
(95 \% \mathrm{Cl})\end{array}$ \\
\hline $\begin{array}{l}\text { Acute kidney injury, no. } \\
(\%)\end{array}$ & $40(45.5 \%)$ & $34(53.1 \%)$ & 0.35 & $\begin{array}{l}0.73 \\
(0.38- \\
1.40)\end{array}$ \\
\hline \multicolumn{5}{|l|}{ Infection sites, no. (\%) } \\
\hline Pulmonary infection & $64(72.7 \%)$ & $50(78.1 \%)$ & 0.45 & $\begin{array}{l}0.74 \\
(0.35- \\
1.59)\end{array}$ \\
\hline Bloodstream infection & $35(39.8 \%)$ & $10(15.6 \%)$ & 0.001 & $\begin{array}{l}3.56 \\
(1.60- \\
7.92)\end{array}$ \\
\hline Urinary tract infection & $26(29.5 \%)$ & $16(25.0 \%)$ & 0.53 & $\begin{array}{l}1.25 \\
(0.61- \\
2.60)\end{array}$ \\
\hline $\begin{array}{l}\text { Gastrointestinal } \\
\text { infection }\end{array}$ & $21(23.9 \%)$ & $16(25.0 \%)$ & 0.87 & $\begin{array}{l}0.94 \\
(0.45- \\
1.99)\end{array}$ \\
\hline Other sites infections & 37 (42.0\%) & $25(39.1 \%)$ & 0.71 & $\begin{array}{l}1.13 \\
(0.58- \\
2.18)\end{array}$ \\
\hline \multicolumn{5}{|l|}{ CRGNB types, no. (\%) } \\
\hline CRKP & $38(43.2 \%)$ & $22(34.4 \%)$ & 0.27 & $\begin{array}{l}1.45 \\
(0.74- \\
2.82)\end{array}$ \\
\hline CRPA & 31 (35.2\%) & $25(39.1 \%)$ & 0.63 & $\begin{array}{l}0.84 \\
(0.43- \\
1.65)\end{array}$ \\
\hline CRAB & $17(19.3 \%)$ & $15(23.4 \%)$ & 0.54 & $\begin{array}{l}0.78 \\
(0.35- \\
1.71)\end{array}$ \\
\hline Other CRE & $2(2.3 \%)$ & $2(3.1 \%)$ & 1.00 & $\begin{array}{l}0.72 \\
(0.10- \\
5.25)\end{array}$ \\
\hline \multicolumn{5}{|l|}{ Antibiotic therapy, no. (\%) } \\
\hline $\begin{array}{l}\text { Combinations with } \\
\text { Tigecycline }\end{array}$ & $25(28.4 \%)$ & $14(21.8 \%)$ & 0.36 & $\begin{array}{l}1.41 \\
(0.67- \\
3.01)\end{array}$ \\
\hline $\begin{array}{l}\text { Combinations with } \\
\text { Polymyxin B }\end{array}$ & $14(15.9 \%)$ & $10(15.6 \%)$ & 0.96 & $\begin{array}{l}1.02 \\
(0.42- \\
2.47)\end{array}$ \\
\hline
\end{tabular}




\begin{tabular}{|c|c|c|c|c|}
\hline & $\begin{array}{l}\text { Empirically sensitive } \\
\text { antibiotic therapy groups, } \mathrm{N} \\
=88\end{array}$ & $\begin{array}{l}\text { Standard antibiotic } \\
\text { therapy groups, } N=64\end{array}$ & $\mathbf{P}$ & $\begin{array}{l}\text { OR } \\
(95 \% \mathrm{Cl})\end{array}$ \\
\hline $\begin{array}{l}\text { Ceftazidime- } \\
\text { avibactam } \\
\text { monotherapy }\end{array}$ & $13(14.7 \%)$ & $4(6.2 \%)$ & 0.10 & $\begin{array}{l}2.60 \\
(0.81- \\
8.39)\end{array}$ \\
\hline Other therapies & 36 (40.9\%) & 36 (56.3\%) & 0.06 & $\begin{array}{l}0.53 \\
(0.28- \\
1.03)\end{array}$ \\
\hline $\begin{array}{l}\text { Duration from } \\
\text { colonization to } \\
\text { infection, day }\end{array}$ & 13.5 & 12.8 & 0.54 & / \\
\hline $\begin{array}{l}\text { Length of ICU stay after } \\
\text { infection, day }\end{array}$ & 16.7 & 16.8 & 0.61 & / \\
\hline $\begin{array}{l}\text { Length of hospital stay } \\
\text { after infection, day }\end{array}$ & 32.8 & 24.2 & 0.22 & / \\
\hline Death, no. (\%) & $15(17.0 \%)$ & 24 (37.5\%) & 0.004 & $\begin{array}{l}0.34 \\
(0.16- \\
0.73)\end{array}$ \\
\hline
\end{tabular}

\section{Discussion:}

Our study found that CRGNB-colonized patients who underwent active surveillance on ICU admission had a higher risk of infection and confirmed that empirically sensitive antibiotic therapy could effectively lower patient mortality.

Contact isolation and hand hygiene have been widely emphasized, while much controversy surrounds the implementation of active surveillance for its questionable cost-effectiveness(17). CRGNB colonization has increased with the widespread prevalence of CRGNB in Asia. Indeed, at clinical level, the medical personnel are often overwhelmed by the concomitant increase in workload following the implementation of basic IPC measures. In some areas with limited resources, active surveillance is not even recommended for asymptomatic patients(17).

Clinical studies have consistently shown that CRGNB colonization is a high-risk factor for infection(18, 19). However, the risk factors of CRGNB infection after colonization are not necessarily the same for CRGNB colonization which may also depend on the patient's immune status. It is widely acknowledged that advanced age, serious disease status, including higher sequential organ failure assessment (SOFA), Pitt score, Charlson score, carbapenem drug exposure, and invasive catheterization are high-risk factors for MDR infection after colonization(19, 20).

For CRGNB-colonized patients, a series of stringent measures were adopted, including contact isolation and strict hand hygiene to avoid cross-infection; nonetheless, CRGNB infection after colonization was 
unavoidable, leading to a poor patient prognosis. Addressing risk factors of infection after colonization earlier may potentially be the solution to this conundrum. Herein, CRKP and CRPA colonization were associated with greater CRGNB infection rates than CRAB and other CRE. Other identified risk factors included invasive catheterization, history of carbapenem use, and immunocompromise. It is important to note that the high-risk factors analysis does not accurately predict which patients will develop CRGNB infection. In this regard, many scoring systems based on high-risk factors have been designed to solve this problem. However, no consensus has been reached on the accuracy of these scoring systems due to the inherent differences in regional characteristics, medical habits, and types of diseases(19-21). Building a more efficient and robust prediction model by artificial intelligence may potentially be the solution. SDD programs have been implemented in some European countries to curb CRGNB infection rates after CRE colonization; however, the efficiency is uncertain due to their implementation in low CRE epidemic areas(17). Given the uncertainty of the effects of SDD, our center did not adopt any decontamination strategy.

An increasing body of evidence suggests that early empirical antibiotic use can reduce mortality of severe sepsis or severe pneumonia $(22,23)$. The Infectious Diseases Society of America (IDSA) recommends that empirical antibiotics use should take it into account of antibiotic sensitivity data in the past six months and antibiotic exposure in the past 30 days (2). The European consensus recommends that the empirical treatment of CRGNB should be limited to critically ill patients, and it is necessary to know the drug sensitivity of CRGNB to carry out empirical treatment(13). In addition, misuse of antibiotics can also be dangerous. Elena Carrara et al. pointed out that inappropriate empirical antibiotic use was closely related to mortality MDR strain infections (24).

Interestingly, in a randomized controlled study by Yael Zak-Doron et al., the mortality rate was not reduced in patients with severe CRGNB infection that received early (during the first 48 hours after culture taking) empirical antibiotic treatments with mainly colistin (96\%)(25). This conclusion is surprising since $77 \%$ of CRGNB infections were related to $C R A B$, and all patients received empirical antibiotic treatment. If empirical treatment can prevent early death, this exclusion may bias the results to be ineffective, but unfortunately, the study does not have complete data on early death and its empirical treatment(25). In addition, the study found that documented colonization $(P=0.044, O R=0.76)$ may be a potential protective factor, which may be related to empirical antibiotic therapy(25). Inappropriate empirical treatment is dangerous and emphasizes the importance of active surveillance. Active surveillance provides more background information on bacterial colonization, although subsequent infections in CRGNB colonized patients are possibly mixed infections or another pathogenic bacterium. However, during severe infection or even septic shock management, microbiological data from CRGNB-colonized patients provides evidence of the potential etiology with drug sensitivity results to help clinicians in the decision-making process(13). Herein, the empirical antibiotic treatments of tigecycline, colistin, ceftazidime-avibactam achieved good results.

There were some limitations in our research. This study was a single-center prospective observational study, and the level of evidence was low. To substantiate our findings, more studies should be conducted 
involving multiple centers and clinical trials. Moreover, potential confounding factors such as changes in recommendations for CRGNB treatment and clinical experience of physicians were not taken into account.

\section{Conclusions:}

Colonized patients are prone to develop an infection with CRGNB; CRKP and CRPA colonization, immunocompromise, invasive procedures, and history of carbapenem use were identified as risk factors. Once the infection is diagnosed, empirical antibiotic treatments can be used to reduce mortality rates.

\section{Abbreviations}

CRGNB

Carbapenem-resistant Gram-negative Bacteria

CRE

carbapenem-resistant enterobacteria

ICU

intensive care unit (ICU)

IPC

infection prevention and control

MDR

multiple drug resistance

CRKP

carbapenem-resistant Klebsiella pneumoniae

CRPA

carbapenem-resistant Pseudomonas aeruginosa

CRAB

carbapenem-resistant Acinetobacter baumannii

MIC

Minimum inhibitory concentration

CLSI

Clinical and Laboratory Standards Institute

OR

Odds ratio

$\mathrm{Cl}$

confidence interval

APACHE

acute physiology and chronic health evaluation

SOFA

sequential organ failure assessment 
SDD

selective digestive decontamination

\section{Declarations}

Acknowledgements: Ethics approval and consent to participate: Our research has been approved by the ethics committee of the second affiliated hospital of Zhejiang University School of Medicine.

Informed consent: The study has obtained informed consent from patients in the observation cohort.

Consent for publication: Yes.

Availability of data and materials: The data that support the findings of this study are available from Man Huang but restrictions apply to the availability of these data, which were used under license for the current study, and so are not publicly available.

Competing interests: The authors declare that they have no competing interests.

Funding: Not applicable.

Authors' contributions: Qiqiang Liang and Juan Chen collected and analyzed the data and wrote the first draft of the manuscript. Juan Chen assists in the collection of information. Yongshan Xu and Yibing Chen assisted with the statistical analyses of the microorganisms. Man Huang reviewed and modified the draft of the manuscript.

Acknowledgements: Thanks to the clinicians in the general ICU for their support and guidance in data collection.

\section{References}

1. Willyard C. The drug-resistant bacteria that pose the greatest health threats. Nature. 2017;543(7643):15.

2. Tamma PD, Aitken SL, Bonomo RA, Mathers AJ, van Duin D, Clancy CJ. Infectious Diseases Society of America Guidance on the Treatment of Extended-Spectrum beta-lactamase Producing Enterobacterales (ESBL-E), Carbapenem-Resistant Enterobacterales (CRE), and Pseudomonas aeruginosa with Difficult-to-Treat Resistance (DTR-P. aeruginosa). Clin Infect Dis. 2021;72(7):110916.

3. Karampatakis T, Tsergouli K, losifidis E, Antachopoulos C, Karapanagiotou A, Karyoti A, et al. Impact of active surveillance and infection control measures on carbapenem-resistant Gram-negative bacterial colonization and infections in intensive care. J Hosp Infect. 2018;99(4):396-404.

4. Gandra S, Ellison RT 3. Modern trends in infection control practices in intensive care units. J Intensive Care Med. 2014;29(6):311-26. rd. . 
5. Liang Q, Yan C, Xu Z, Huang M. Preemptive isolation and active surveillance in the prevention and control of nosocomial infection reduce the incidence of carbapenem-resistant Enterobacteriaceae. Infect Dis (Lond). 2019;51(5):377-9.

6. Tang SSL, Chee E, Teo JQ, Chlebicki MP, Kwa ALH. Incidence of a subsequent carbapenem-resistant Enterobacteriaceae infection after previous colonisation or infection: a prospective cohort study. (1872-7913 (Electronic)).

7. Asensio A, Cantero M, Shaw E, Vergara-Lopez S. Control strategies for carbapenemase-producing Enterobacteriaceae at different levels of the healthcare system. Enfermedades infecciosas y microbiologia clinica. 2014;32(Suppl 4):61-6.

8. Niederman MS, Alder J, Bassetti M, Boateng F, Cao B, Corkery K, et al. Inhaled amikacin adjunctive to intravenous standard-of-care antibiotics in mechanically ventilated patients with Gram-negative pneumonia (INHALE): a double-blind, randomised, placebo-controlled, phase 3, superiority trial. Lancet Infect Dis. 2020;20(3):330-40.

9. Xu F, He LL, Che LQ, Li W, Ying SM, Chen ZH, et al. Aerosolized antibiotics for ventilator-associated pneumonia: a pairwise and Bayesian network meta-analysis. Crit Care. 2018;22(1):301.

10. De Waele JJ, Martin-Loeches I. Optimal duration of antibiotic treatment in Gram-negative infections. Curr Opin Infect Dis. 2018;31(6):606-11.

11. Tumbarello M, Trecarichi EM, De Rosa FG, Giannella M, Giacobbe DR, Bassetti M, et al. Infections caused by KPC-producing Klebsiella pneumoniae: differences in therapy and mortality in a multicentre study. J Antimicrob Chemother. 2015;70(7):2133-43.

12. Gutierrez-Gutierrez B, Salamanca E, de Cueto M, Hsueh PR, Viale P, Pano-Pardo JR, et al. Effect of appropriate combination therapy on mortality of patients with bloodstream infections due to carbapenemase-producing Enterobacteriaceae (INCREMENT): a retrospective cohort study. Lancet Infectious Diseases. 2017;17(7):726-34.

13. Bedos JP, Daikos G, Dodgson AR, Pan A, Petrosillo N, Seifert H, et al. Early identification and optimal management of carbapenem-resistant Gram-negative infection. J Hosp Infect. 2021;108:158-67.

14. Kalil AC, Metersky ML, Klompas M, Muscedere J, Sweeney DA, Palmer LB, et al. Management of Adults With Hospital-acquired and Ventilator-associated Pneumonia: 2016 Clinical Practice Guidelines by the Infectious Diseases Society of America and the American Thoracic Society. (15376591 (Electronic)).

15. Hooton TM, Bradley Sf Fau - Cardenas DD, Cardenas Dd Fau - Colgan R, Colgan R, Fau - Geerlings SE. Geerlings Se Fau - Rice JC, Rice Jc Fau - Saint S, et al. Diagnosis, prevention, and treatment of catheter-associated urinary tract infection in adults: 2009 International Clinical Practice Guidelines from the Infectious Diseases Society of America. (1537-6591 (Electronic)).

16. Khwaja A. KDIGO clinical practice guidelines for acute kidney injury. Nephron Clin Pract. 2012;120(4):c179-84.

17. Thatrimontrichai A, Apisarnthanarak A. Active surveillance culture program in asymptomatic patients as a strategy to control multidrug-resistant gram-negative organisms: What should be considered? J 
Formos Med Assoc. 2020;119(11):1581-5.

18. Tischendorf J, de Avila RA, Safdar N. Risk of infection following colonization with carbapenemresistant Enterobactericeae: A systematic review. Am J Infect Control. 2016;44(5):539-43.

19. Burillo A, Munoz P, Bouza E. Risk stratification for multidrug-resistant Gram-negative infections in ICU patients. Curr Opin Infect Dis. 2019;32(6):626-37.

20. Song JY, Jeong IS. Validation of a carbapenem-resistant Enterobacteriaceae colonization risk prediction model: A retrospective cohort study in Korean intensive care units. (1527-3296 (Electronic)).

21. Goodman KA-OX, Simner PJ, Klein EY, Kazmi AQ, Gadala A, Toerper MF, et al. Predicting probability of perirectal colonization with carbapenem-resistant Enterobacteriaceae (CRE) and other carbapenemresistant organisms (CROs) at hospital unit admission. (1559-6834 (Electronic)).

22. Metlay JP, Waterer GW, Long AC, Anzueto A, Brozek J, Crothers K, et al. Diagnosis and Treatment of Adults with Community-acquired Pneumonia. An Official Clinical Practice Guideline of the American Thoracic Society and Infectious Diseases Society of America. Am J Respir Crit Care Med. 2019;200(7):e45-67.

23. Singer M, Deutschman CS, Seymour CW, Shankar-Hari M, Annane D, Bauer M, et al. The Third International Consensus Definitions for Sepsis and Septic Shock (Sepsis-3). JAMA. 2016;315(8):801-10.

24. Carrara E, Pfeffer I, Zusman O, Leibovici L, Paul M. Determinants of inappropriate empirical antibiotic treatment: systematic review and meta-analysis. (1872-7913 (Electronic)).

25. Zak-Doron Y, Dishon Benattar Y, Pfeffer I, Daikos GL, Skiada A, Antoniadou A, et al. The Association Between Empirical Antibiotic Treatment and Mortality in Severe Infections Caused by Carbapenemresistant Gram-negative Bacteria: A Prospective Study. Clin Infect Dis. 2018;67(12):1815-23.

\section{Figures}




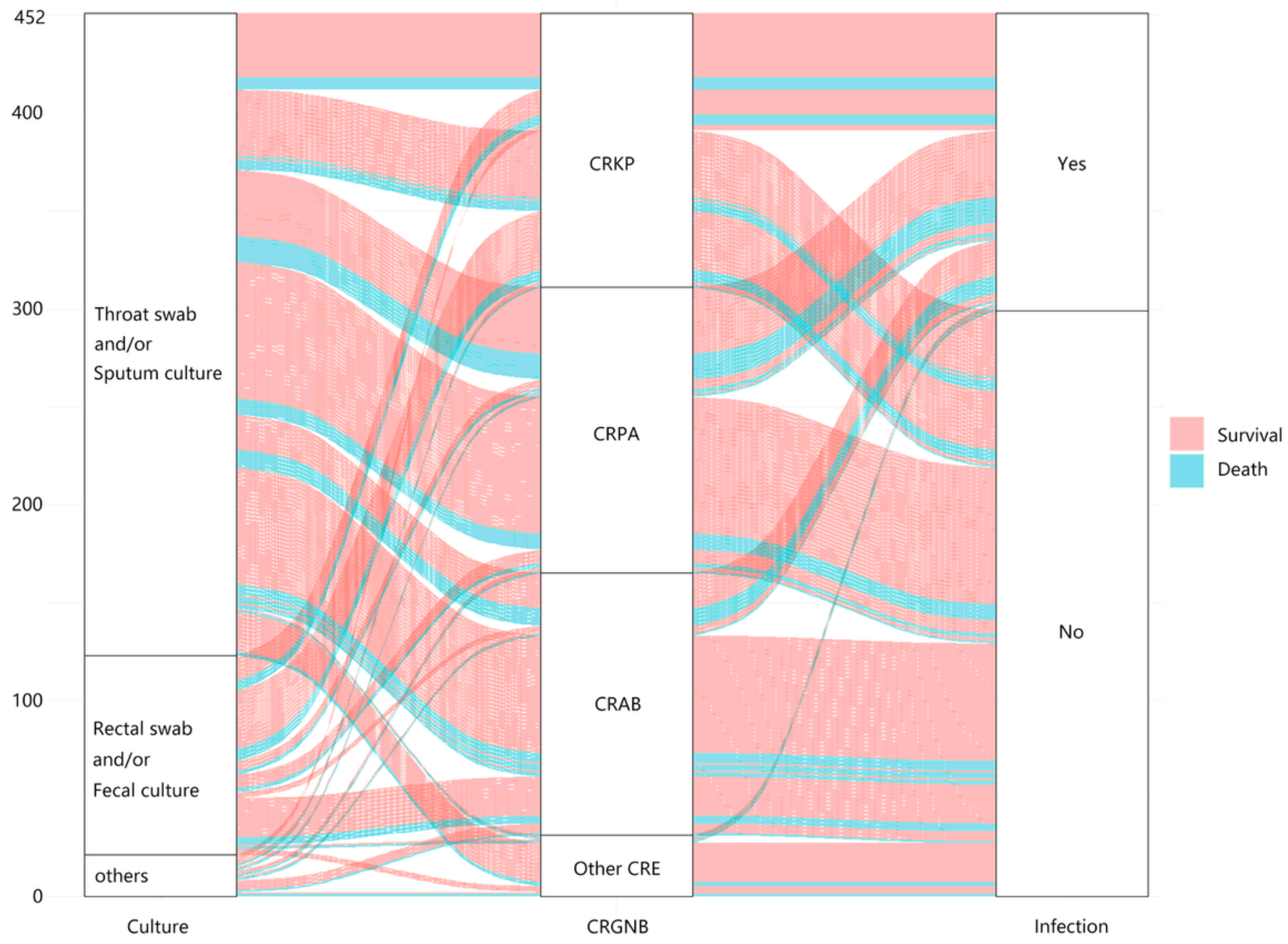

Figure 1

Alluvial diagram of the feature distribution in CRGNB colonization patients by active surveillance. 
Kaplan-Meier curve of CRGNB infection patients after colonization with different timing of antibiotics

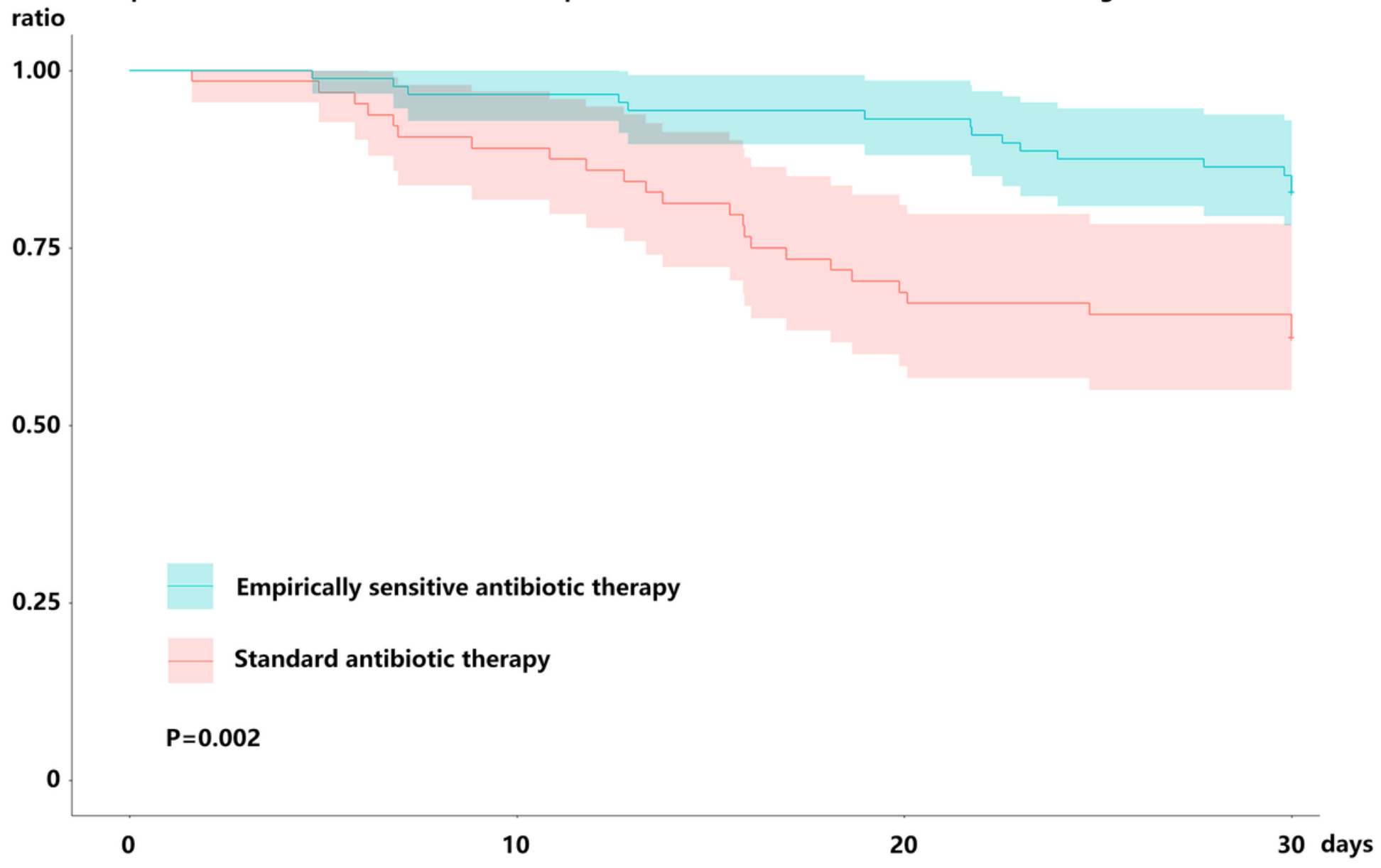

Figure 2

Kaplan-Meier curve of CRGNB infection patients after colonization with different timing of antibiotics. 